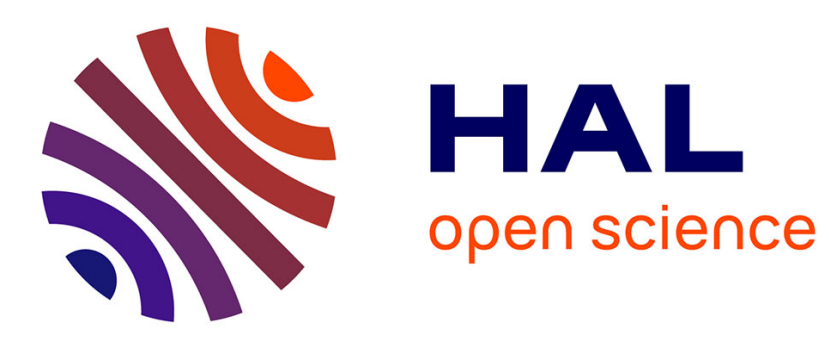

\title{
An analog to the Schur-Siegel-Smyth trace problem
}

Valérie Flammang

\section{To cite this version:}

Valérie Flammang. An analog to the Schur-Siegel-Smyth trace problem. Mathematics of Computation, 2020, 89, pp.2387-2398. 10.1090/mcom/3518 . hal-03295898

\section{HAL Id: hal-03295898 \\ https://hal.science/hal-03295898}

Submitted on 22 Jul 2021

HAL is a multi-disciplinary open access archive for the deposit and dissemination of scientific research documents, whether they are published or not. The documents may come from teaching and research institutions in France or abroad, or from public or private research centers.
L'archive ouverte pluridisciplinaire HAL, est destinée au dépôt et à la diffusion de documents scientifiques de niveau recherche, publiés ou non, émanant des établissements d'enseignement et de recherche français ou étrangers, des laboratoires publics ou privés. 


\title{
AN ANALOG TO THE SCHUR-SIEGEL-SMYTH TRACE PROBLEM
}

\author{
V. FLAMMANG
}

\begin{abstract}
If $\alpha$ denotes a totally positive algebraic integer of degree $d$ i.e., its conjugates $\alpha_{1}=\alpha, \ldots, \alpha_{d}$ are positive real numbers, we define $\mathrm{S}_{k}(\alpha)=\sum_{i=1}^{d} \alpha_{i}^{k} . \mathrm{S}_{1}(\alpha)$ is the usual trace of $\alpha$ and was studied by many authors throughout the years. In this paper, we focus our attention on the values of $S_{2}(\alpha)$ and our purpose is to establish for $S_{2}$ the same kind of results than for the trace.
\end{abstract}

\section{Introduction}

Let $\alpha$ be a totally positive algebraic integer of degree $d \geq 2$ i.e all its conjugates $\alpha_{1}=\alpha, \ldots, \alpha_{d}$ are positive real numbers. For $k \geq 1$, we put

$$
\mathrm{S}_{k}(\alpha)=\sum_{i=1}^{d} \alpha_{i}^{k}
$$

Then, $\mathrm{S}_{1}(\alpha)=\operatorname{Tr}(\alpha)$ is the usual trace of $\alpha$ and $\operatorname{tr}(\alpha)=\frac{1}{d} \operatorname{Tr}(\alpha)$ denotes the absolute trace of $\alpha$. The "Schur-Siegel-Smyth trace problem" is the following: fix $\rho<2$, then show that all but finitely many totally positive algebraic integers $\alpha$ have $\operatorname{tr}(\alpha)>\rho$. Many authors studied this problem. All the results after those of I. Schur [Sc] and C.L. Siegel [Si] are based on the method of explicit auxiliary functions. For more details on this problem, see for example [S1], [ABP], [AP1], [AP2],[AP3], [LW], [DW], [F1]. In 2016, we solved it for $\rho<1.792812$ which is the better result at our knowledge [F1]. On the other hand, J.P. Serre [AP3] showed that this method does not give such an inequality for any $\rho$ larger than $1.8983021 \ldots$. Therefore this method cannot be used to prove that 2 is the smallest limit point of the set of quantities $\{\operatorname{tr}(\alpha), \alpha$ totally positive algebraic integer $\}$. In this paper, we call the $\mathrm{S}_{2}$ measure of $\alpha$ the quantity $\mathrm{S}_{2}(\alpha)=\sum_{i=1}^{d} \alpha_{i}^{2}$ and the absolute $\mathrm{S}_{2}$ measure of $\alpha$ is defined by $\mathrm{s}_{2}(\alpha)=\frac{1}{d} \mathrm{~S}_{2}(\alpha) . \mathcal{S}_{2}$ denotes the set of all $\mathrm{s}_{2}(\alpha)$ (where $\alpha$ is a totally positive algebraic integer) . Like for the trace, our aim is to search the supremum of all $\rho>0$ such that all but finitely many totally positive algebraic integers satisfy $\mathrm{s}_{2}(\alpha)>\rho$. First, we prove that $\rho \leq 6$. We consider the following polynomial due to C. L. Siegel [Si]:

$$
F(X)=\prod_{k=1}^{\frac{p-1}{2}}\left(X-\left(\zeta^{k}+\zeta^{-k}+2\right)\right)
$$

where $\zeta$ is a primitive $p$ th root of unity for $p$ an odd prime. We have

$$
\mathrm{S}_{2}(F)=\sum_{k=1}^{\frac{p-1}{2}}\left(\left(\zeta^{k}\right)^{2}+\left(\zeta^{-k}\right)^{2}\right)+6 \sum_{k=1}^{\frac{p-1}{2}} 1+4 \sum_{k=1}^{\frac{p-1}{2}}\left(\zeta^{k}+\zeta^{-k}\right)=6 \frac{p-1}{2}-5 .
$$

Hence, $\mathrm{s}(F)=6-\frac{10}{p-1}$. Therefore, the analog of the "Schur-Siegel-Smyth trace problem" for the $\mathrm{S}_{2}$ measure is: Fix $\rho<6$. Then prove that all but finitely many totally positive algebraic integers $\alpha$ have $\mathrm{s}_{2}(\alpha)>\rho$. Y. Liang and Q. Wu [LW] in 2011 solved the problem for $\rho<5.31935$. In this paper, we solve it for $\rho<5.321767$. More precisely, using the method of explicit auxiliary functions, we prove the following result: 
Theorem 1. If $\alpha$ is a totally positive algebraic integer of degree $d$ whose minimal polynomial is different from $x-1, x-2, x^{2}-3 x+1, x^{3}-5 x^{2}+6 x-1, x^{4}-7 x^{3}+14 x^{2}-8 x+1$, $x^{5}-9 x^{4}+28 x^{3}-35 x^{2}+15 x-1$ et $x^{6}-11 x^{5}+45 x^{4}-84 x^{3}+70 x^{2}-21 x+1$ then we have:

$$
\mathrm{s}_{2} \geq 5.321767 \text {. }
$$

Corollary 1. The seven first points of $\mathcal{S}_{2}$ are:

$$
\begin{array}{ll}
1 & =\mathrm{s}_{2}(x-1) \\
3.5 & =\mathrm{s}_{2}\left(x^{2}-3 x+1\right) \\
4 & =\mathrm{s}_{2}(x-2) \\
4.333333 \ldots & =\mathrm{s}_{2}\left(x^{3}-5 x^{2}+6 x-1\right) \\
5 & =\mathrm{s}_{2}\left(x^{5}-9 x^{4}+28 x^{3}-35 x^{2}+15 x-1-\right. \\
5.166666 \ldots & =\mathrm{s}_{2}\left(x^{6}-11 x^{5}+45 x^{4}-84 x^{3}+70 x^{2}-21 x+1\right) \\
5.25 & =\mathrm{s}_{2}\left(x^{4}-7 x^{3}+14 x^{2}-8 x+1\right)
\end{array}
$$

We recall that an algebraic integer $\alpha$ is reciprocal if $\alpha$ and $\frac{1}{\alpha}$ are conjugates.

Corollary 2. If $\alpha$ is a totally positive reciprocal algebraic integer of degree $d$ whose minimal polynomial is different from $x^{2}-3 x+1, x^{2}-4 x+1, x^{4}-7 x^{3}+13 x^{2}-7 x+1$ and $x^{6}-11 x^{5}+$ $41 x^{4}-63 x^{3}+41 x^{2}-11 x+1$, then we have :

$$
\mathrm{s}_{2}(\alpha) \geq 7.2465075 \text {. }
$$

Finally, we follow the method developed by J. P. Serre (see Appendix B in [AP3]) for the trace to prove the following result:

Theorem 2. The method of auxiliary functions does not give an inequality involved in Theorem 1 for any $\rho$ larger than 5.895237 .... Therefore this method cannot be used to find a limit point greater than this constant. We can not prove by this way that 6 is the smallest limit point of $\mathcal{S}_{2}$.

\section{Proof of Theorem 1}

\subsection{The principle of auxiliary functions}

The auxiliary function involved in the study of the $\mathrm{S}_{2}$ measure is of the following type:

$$
\text { for } x>0, f(x)=x^{2}-\sum_{1 \leq j \leq J} c_{j} \log \left|Q_{j}(x)\right|
$$

where the $c_{j}$ are positive real numbers and the polynomials $Q_{j}$ are nonzero polynomials in $\mathbb{Z}[x]$. Let $m$ be the minimum of the function $f$. If $P$ does not divide any $Q_{j}$, then we have

$$
\sum_{i=1}^{d} f\left(\alpha_{i}\right) \geq m d
$$

i.e.,

$$
\mathrm{S}_{2}(\alpha) \geq m d+\sum_{1 \leq j \leq J} c_{j} \log \left|\prod_{i=1}^{d} Q_{j}\left(\alpha_{i}\right)\right| .
$$


Since $P$ is monic and does not divide any $Q_{j}$ then $\prod_{i=1}^{d} Q_{j}\left(\alpha_{i}\right)$ is a nonzero integer because it is the resultant of $P$ and $Q_{j}$.

Hence, if $\alpha$ is not a root of $Q_{j}$, we have

$$
\mathrm{s}_{2}(\alpha) \geq m
$$

Remark: The following sections reproduce the corresponding sections of [F1].

\subsection{Link between auxiliary functions and generalized integer transfinite di- ameter}

Let $K$ be a compact subset of $\mathbb{C}$. The transfinite diameter of $K$ is defined by

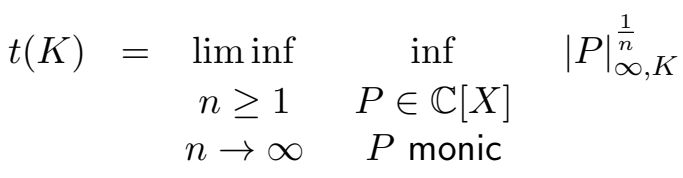

$$
\begin{aligned}
& \operatorname{deg}(P)=n
\end{aligned}
$$

where $|P|_{\infty, K}=\sup _{z \in K}|P(z)|$ for $P \in \mathbb{C}[X]$.

We define the integer transfinite diameter of $K$ by

$$
\begin{aligned}
& t_{\mathbb{Z}}(K)=\liminf _{n>1} \quad \inf _{P \in \mathbb{Z}[X]}|P|_{\infty, K}^{\frac{1}{n}} \\
& n \rightarrow \infty \quad \operatorname{deg}(P)=n
\end{aligned}
$$

Finally, if $\varphi$ is a positive function defined on $K$, the $\varphi$-generalized integer transfinite diameter of $K$ is defined by

$$
\begin{array}{cccc}
t_{\mathbb{Z}, \varphi}(K)= & \liminf & \inf & \sup \\
& n \geq 1 & P \in \mathbb{Z}[X] \quad z \in K \\
& n \rightarrow \infty & \operatorname{deg}(P)=n
\end{array}
$$

In the auxiliary function (1), we replace the coefficients $c_{j}$ by rational numbers $a_{j} / q$ where $q$ is a positive integer such that $q . c_{j}$ is an integer for all $1 \leq j \leq J$. Then we can write:

$$
\text { for } x>0, f(x)=x^{2}-\frac{t}{r} \log |Q(x)| \geq m
$$

where $Q=\prod_{j=1}^{J} Q_{j}^{a_{j}} \in \mathbb{Z}[X]$ is of degree $r=\sum_{j=1}^{J} a_{j} \operatorname{deg} Q_{j}$ and $t=\sum_{j=1}^{J} c_{j} \operatorname{deg} Q_{j}$ (this formulation was introduced by J. P. Serre). Thus we seek a polynomial $Q \in \mathbb{Z}[X]$ such that

$$
\sup _{x>0}|Q(x)|^{t / r} e^{-x^{2}} \leq e^{-m}
$$

If we suppose that $t$ is fixed, it is equivalent to find an effective upper bound for the weighted integer transfinite diameter over the interval $\left[0, \infty\left[\right.\right.$ with the weight $\varphi(x)=e^{-x^{2}}$ :

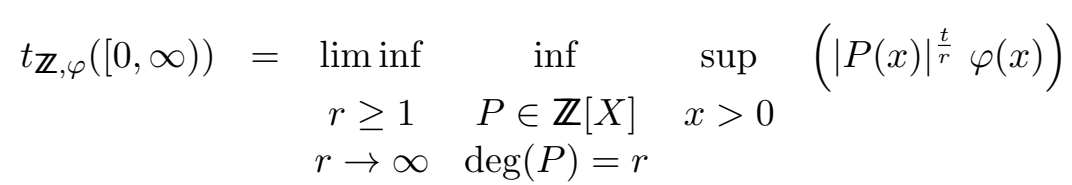

Remark: Even if we have replaced the compact $K$ by the infinite interval $[0, \infty[$, the weight $\varphi$ ensures that the quantity $t_{\mathbb{Z}, \varphi}([0, \infty))$ is finite. 


\subsection{Construction of an auxiliary function}

The main point is to find a set of "good "polynomials $Q_{j}$, i.e., which gives the best possible value for $m$. Until 2003, the polynomials were found heuristically. In 2003, Q. Wu [Wu] developed an algorithm that allows a systematic search of "good "polynomials. His method was the following. We consider an auxiliary function as defined by (1). We fix a set $E_{0}$ of control points, uniformly distributed on the real interval $I=[0, A]$ where $A$ is "sufficiently large ". Thanks to the LLL algorithm, we find a polynomial $Q$ small on $E_{0}$ within the meaning of the quadratic norm. We test this polynomial in the auxiliary function and we keep only the factors of $Q$ which have a nonzero exponent. The convergence of this new function gives local minima that we add to the set of points $E_{0}$ to get a new set of control points $E_{1}$. We use again the LLL algorithm with the set $E_{1}$ and the process is repeated.

In 2006, we made two improvements to this previous algorithm in the use of the LLL algorithm. The first one is, at each step, to take into account not only the new control point but also the new polynomials of the best auxiliary function. The second one is the introduction of a corrective coefficient $t$. The idea is to get good polynomials $Q_{j}$ by induction. Thus, we call this algorithm the recursive algorithm. The first step consists in the optimization of the auxiliary function $f_{1}=x^{2}-t \log x$. We have $t=c_{1}$ where $c_{1}$ is the value that gives the best function $f_{1}$. We suppose that we have some polynomials $Q_{1}, Q_{2}, \ldots, Q_{J}$ and a function $f$ as good as possible for this set of polynomials in the form (2). We seek a polynomial $R \in \mathbb{Z}[x]$ of degree $k$ ( $k=10$ for instance) such that

$$
\sup _{x \in I}|Q(x) R(x)|^{\frac{t}{r+k}} e^{-x^{2}} \leq e^{-m}
$$

where $Q=\prod_{j=1}^{J} Q_{j}$. We want the quantity

$$
\sup _{x \in I}|Q(x) R(x)| \exp \left(\frac{-x^{2}(r+k)}{t}\right)
$$

to be as small as possible. We apply the LLL algorithm to the linear forms

$$
Q\left(x_{i}\right) R\left(x_{i}\right) \exp \left(\frac{-x_{i}{ }^{2}(r+k)}{t}\right) \text {. }
$$

The $x_{i}$ are control points which are points uniformly distributed on the interval $I$ to which we have added points where $f$ has local minima. Thus we find a polynomial $R$ whose irreducible factors $R_{j}$ are good candidates to enlarge the set $\left\{Q_{1}, \ldots, Q_{J}\right\}$. We only keep the factors $R_{j}$ that have a nonzero coefficient in the newly optimized auxiliary function $f$.After optimization, some previous polynomials $Q_{j}$ may have a zero exponent and so are removed.

\subsection{Optimization of the $c_{j}$}

We have to solve a problem of the following form: find

$$
\max _{C} \min _{x \in X} f(x, C)
$$

where $f(x, C)$ is a linear form with respect to $C=\left(c_{0}, c_{1}, \ldots, c_{k}\right)\left(c_{0}\right.$ is the coefficient of $x$ and is equal to 1 ) and $X$ is a compact domain of $\mathbb{C}$, the maximum is taken over $c_{j} \geq 0$ for $j=0, \ldots, k$. A classical solution consists in taking very many control points $\left(x_{i}\right)_{1 \leq i \leq N}$ and in solving the standard problem of linear programming:

$$
\max _{C} \min _{1 \leq j \leq N} f\left(x_{i}, C\right) .
$$

The result depends then on the choice of the control points. 
The idea of the semi infinite linear programming (introduced into Number Theory by C. J. Smyth [S2]) consists in repeating the previous process adding at each step new control points and verifying that this process converges to $m$, the value of the linear form for an optimum choice of $C$. The algorithm is the following:

(1) We choose an initial value for $C$ i.e., $C^{0}$ and we calculate

$$
m_{0}^{\prime}=\min _{x \in X} f\left(x, C^{0}\right) .
$$

(2) We choose a finite set $X_{0}$ of control points belonging to $\mathrm{X}$ and we have

$$
m_{0}^{\prime} \leq m \leq m_{0}=\min _{x \in X_{0}} f\left(x, C^{0}\right) .
$$

(3) We add to $X_{0}$ the points where $f\left(x, C^{0}\right)$ has local minima to get a new set $X_{1}$ of control points.

(4) We solve the usual linear programming problem:

$$
\max _{C} \min _{x \in X_{1}} f(x, C)
$$

We get a new value for $C$ denoted by $C^{1}$ and a result of the linear programming equal to $m_{1}^{\prime}=\min _{x \in X} f\left(x, C^{1}\right)$. Then we have

$$
m_{0}^{\prime} \leq m_{1}^{\prime} \leq m \leq m_{1}=\min _{x \in X_{1}} f\left(x, C^{1}\right) \leq m_{0}
$$

(5) We repeat the steps from (2) to (4) and thus we get two sequences $\left(m_{i}\right)$ and $\left(m_{i}^{\prime}\right)$ which satisfy

$$
m_{0}^{\prime} \leq m_{1}^{\prime} \leq \ldots \leq m_{i}^{\prime} \leq m \leq m_{i} \leq \ldots \leq m_{1} \leq m_{0},
$$

We stop when there is a good enough convergence, for example when $m_{i}-m_{i}^{\prime} \leq 10^{-6}$. Suppose that $p$ iterations are sufficient then we take $m=m_{p}^{\prime}$.

\section{Proof of Corollary 2}

Let $\alpha$ be a totally positive reciprocal algebraic integer of degree $d$ with minimal polynomial $P$. Then there exists a totally positive polynomial $Q$ of degree $d / 2$ satisfying:

$$
P(X)=X^{d / 2} Q\left(X+\frac{1}{X}-2\right)
$$

Let $\alpha_{1}, \ldots, \alpha_{d}$ and $\beta_{1}, \ldots, \beta_{d / 2}$ be the roots of $P$ and $Q$ respectively. Then we have:

$$
\text { for } 1 \leq i \leq d / 2, \beta_{i}=\alpha_{i}+\frac{1}{\alpha_{i}}-2
$$

Hence, $\mathrm{S}_{2}(\alpha)=\sum_{i=1}^{d} \alpha_{i}^{2}=\sum_{i=1}^{d / 2}\left(\alpha_{i}^{2}+\frac{1}{\alpha_{i}^{2}}\right)=\sum_{i=1}^{d / 2}\left(\left(\alpha_{i}+\frac{1}{\alpha_{i}}\right)^{2}-2\right)=\sum_{i=1}^{d / 2}\left(\beta_{i}+2\right)^{2}-d$ i.e., $\mathrm{S}_{2}(\alpha)=\mathrm{S}_{2}(\beta)+4 \mathrm{~S}_{1}(\beta)+d$.

So,

$$
\mathrm{s}_{2}(\alpha)=\frac{\mathrm{S}_{2}(\beta)}{2 \frac{d}{2}}+4 \frac{\mathrm{S}_{1}(\beta)}{2 \frac{d}{2}}+1 \geq \frac{5.321767}{2}+2.1 .792812+1=7.2465075,
$$

since $\beta$ satisfies the hypothesis of Theorem 1 above and those of Theorem 1 in [F1]. 


\section{Proof of Theorem 2}

As said in the Introduction, we follow in this section the work and the notations of J. P . Serre [AP3]. Here, the first inequality is:

$$
\forall x>0, x^{2} \geq c+\frac{t}{p} \log |P(x)|
$$

where $t>0$ and $p=\operatorname{deg} P$. Hence, we have to prove that $c<5.895237$. For $0<\gamma<1$, we put $P(x)=x^{\gamma p} R(x)$ where $q=\operatorname{deg} R=p(1-\gamma)$. We need to assume that $|R(0)| \geq 1$. The inequality (1) can be rewritten as:

$$
x^{2} \geq c+t \gamma \log x+\frac{(1-\gamma) t}{q} \cdot \log |R(x)|
$$

Let $0<a<b$. On the interval $(a, b)$, we choose the measure called "equilibrium distribution "in capacity theory: $d \mu(x)=\frac{1}{\pi} \frac{1}{\sqrt{(b-x)(x-a)}} d x$. Now, put:

$$
x=\tau\left(1+y \sqrt{1-g^{2}}\right), a=\tau\left(1-\sqrt{1-g^{2}}\right) \text { and } b=\tau\left(1+\sqrt{1-g^{2}}\right),
$$

where $\tau>0$ and $0<g<1$. Therefore, $d \mu(x)=\frac{1}{\pi} \frac{1}{\sqrt{1-y^{2}}} d y$.

\subsection{Auxiliary calculus}

We will need some integration formulas:

- $\forall \lambda \in \mathbb{C}, \int_{a}^{b} \log |x-\lambda| d \mu(x) \geq \log L$, where $L$ is the capacity of the interval $(a, b)$ and so is equal to $\frac{b-a}{4}$.

- $\int_{a}^{b} \frac{1}{k} \log |K(x)| d \mu(x) \geq \log L$ where $K$ is a polynomial of degree $k$ and highest coefficient of modulus $\geq 1$.

- $\int_{a}^{b} \log x d \mu(x)=\log L+\log \left(1+\frac{u}{2}+\sqrt{u+\frac{u^{2}}{4}}\right)$ where $u=\frac{a}{L}$.

These three formulas were already in [AP3]. Here, we need to calculate $I=\int_{a}^{b} x^{2} d \mu(x)$.

$I=\frac{\tau^{2}}{\pi} \int_{-1}^{1} \frac{1+y \sqrt{1-g^{2}}}{\sqrt{1-y^{2}}} d y=\frac{\tau^{2}}{\pi}\left[\int_{-1}^{1} \frac{d y}{\sqrt{1-y^{2}}}+2 \sqrt{1-g^{2}} \int_{-1}^{1} \frac{y d y}{\sqrt{1-g^{2}}}+\left(1-g^{2}\right) \int_{-1}^{1} \frac{y^{2}}{\sqrt{1-y^{2}}}\right]$.

Then, we put $y=\sin \varphi$ and we obtain $I=\tau^{2}\left(\frac{3-g^{2}}{2}\right)$. Besides, if we put $z=1+\frac{u}{2}+\sqrt{u+\frac{u^{2}}{4}}$,

we have $a=L\left(z+\frac{1}{z}-2\right)$ and $b=4 L+a$ so $\tau=\frac{a+b}{2}=L\left(z+\frac{1}{z}\right)$ and finally we get

$$
\int_{a}^{b} x^{2} d \mu(x)=L^{2}\left(z+\frac{1}{z}\right)^{2}\left(\frac{3-g^{2}}{2}\right) \text {. }
$$

We also need to calculate $J=\int_{a}^{b} \frac{1}{x^{2}} d \mu(x)=\frac{1}{\pi \tau^{2}} \int_{-1}^{1} \frac{1}{\left(1+y \sqrt{1-g^{2}}\right)^{2}} \frac{d y}{\sqrt{1-y^{2}}}$. First, we put $y=\cos \varphi$. Hence, $J=\frac{1}{\pi \tau^{2}\left(1-g^{2}\right)} \int_{0}^{\pi} \frac{d \varphi}{C+\cos \varphi}$ where $C=\frac{1}{\sqrt{1-g^{2}}}>1$. Then, we put $\cos \varphi=\frac{1-v^{2}}{1+v^{2}}$. We get

$J=\frac{1}{\pi \tau^{2}\left(1-g^{2}\right)} \int_{0}^{+\infty} \frac{v^{2}+1}{\left((C-1) v^{2}+(C+1)\right)^{2}} d v=\frac{1}{\pi \tau^{2}\left(1-g^{2}\right)}\left[\frac{-v}{\left(C^{2}-1\right)\left((C-1) v^{2}+(C+1)\right)}+\right]_{0}^{+\infty}$ 
$+\frac{1}{\pi \tau^{2}\left(1-g^{2}\right)}\left[\frac{2 C \arctan \left(\frac{(C-1) v}{\sqrt{C^{2}-1}}\right)}{\left(C^{2}-1\right)^{3 / 2}}\right]_{0}^{+\infty}=\frac{C}{\tau^{2}\left(1-g^{2}\right)\left(C^{2}-1\right)^{3 / 2}}=\frac{1}{g} \frac{1}{\tau^{2} g^{2}}$. But, we have $\tau^{2} g^{2}=$ $a b=a(4 L+a)=L\left(z+\frac{1}{z}-2\right)\left(4 L+L\left(z+\frac{1}{z}-2\right)\right)=L^{2}\left(z-\frac{1}{z}\right)^{2}$. Finally, we get

$$
\int_{a}^{b} \frac{1}{x^{2}} d \mu(x)=\frac{1}{L^{2}\left(z-\frac{1}{z}\right)^{2}}
$$

\subsection{First integration over the interval $(a, b)$}

We begin to integrate the inequality $(2)$ over an interval $(a, b)$ and get:

$$
L^{2}\left(z+\frac{1}{z}\right)^{2}\left(\frac{3-g^{2}}{2}\right) \leq c+t \gamma(\log L+\log z)+t(1-\gamma) \log L
$$

i.e.,

$$
c \leq \underbrace{L^{2}\left(z+\frac{1}{z}\right)^{2}\left(\frac{3-g^{2}}{2}\right)-t \gamma \log z-t \log L}_{f(L, z)}
$$

In order to optimize the function $f(L, z)$, we calculate its partial derivatives to find the pair $(L, z)$ for which they both are zero ( for a given pair $(\gamma, t)$ ). We obtain:

$$
L^{2}=\frac{t\left(1-\gamma^{2}\right)}{4\left(3-g^{2}\right)} \quad \text { and } \quad z=\sqrt{\frac{1+\gamma}{1-\gamma}}
$$

Now, remember that $a=\tau\left(1-\sqrt{1-g^{2}}\right)=L\left(z+\frac{1}{z}-2\right)=\sqrt{\frac{2 t}{3-g^{2}}}\left(1-\sqrt{1-\gamma^{2}}\right)$. Hence, we choose $g=\gamma$ and $\tau=\sqrt{\frac{2 t}{3-g^{2}}}$. Finally, we have

$$
\frac{c}{t} \leq \underbrace{\frac{1}{2}-\frac{1}{2} \log t-\frac{1+\gamma}{2} \log (1+\gamma)-\frac{1-\gamma}{2} \log (1-\gamma)+\frac{1}{2} \log 4\left(3-\gamma^{2}\right)}_{a(\gamma, t)} .
$$

\subsection{Second integration over the interval $(a, b)$}

Let $Q$ be the reciprocal polynomial of the polynomial $R$ i.e., $Q(x)=x^{q} R\left(\frac{1}{x}\right), \operatorname{deg} Q=\operatorname{deg} R=q$. As we assumed that $|R(0)| \geq 1$ then the highest coefficient of the polynomial $Q$ has modulus $\geq 1$. We write the inequality $(2)$ for $\frac{1}{x}$ :

$$
\frac{1}{x^{2}} \geq c+t \gamma \log \frac{1}{x}+\frac{t(1-\gamma)}{q} \log \left|x^{-q} Q(x)\right|,
$$

i.e.,

$$
\frac{1}{x^{2}} \geq c-t \log x+\frac{t(1-\gamma)}{q} \log |Q(x)|
$$

Again, we integrate this inequality over an interval $(a, b)$ and get:

$$
\frac{1}{\gamma} \frac{1}{L^{2}\left(z+\frac{1}{z}\right)^{2}} \leq c-t(\log L+\log z)+t(1-\gamma) \log L
$$

i.e.,

$$
c \leq \underbrace{\frac{1}{\gamma} \frac{1}{L^{2}\left(z+\frac{1}{z}\right)^{2}}+t \gamma \log L+t \log z}_{g(L, z)} .
$$


As before, we want to optimize the function $g(L, z)$. Its partial derivatives are both zero for the following values of $L$ and $z$ :

$$
L^{2}=\frac{1-\gamma^{2}}{2 t \gamma^{4}} \quad \text { and } \quad z=\sqrt{\frac{1+\gamma}{1-\gamma}}
$$

Therefore, we have

$$
\frac{c}{t} \leq \underbrace{\frac{\gamma}{2}-\frac{1-\gamma}{2} \log (1-\gamma)+\frac{1+\gamma}{2} \log (1+\gamma)-\frac{\gamma}{2} \log \left(2 t \gamma^{4}\right)}_{b(\gamma, t)} .
$$

\subsection{The last calculus}

$\mathrm{k}$

Now, we have to find the maximum of the function $c(\gamma, t)=t \inf (a(\gamma, t), b(\gamma, t))$ where $t>0$ and $0<\gamma<1$.

- We put $h(\gamma, t)=t a(\gamma, t)$ and we calculate its partial derivatives:

$$
\frac{\partial h}{\partial \gamma}=\frac{t}{2} \underbrace{\left(-\log (1+\gamma)+\log (1-\gamma)-\frac{2 \gamma}{3-\gamma^{2}}\right)}_{<0} .
$$

Thus, it is no need to compute the other partial derivative to check that they cannot be both zero.

- We put $k(\gamma, t)=t b(\gamma, t)$ and we calculate its partial derivatives:

$$
\frac{\partial k}{\partial \gamma}=\frac{t}{2}\left(-1+\log (1-\gamma)+\log (1+\gamma)-\log \left(2 t \gamma^{4}\right)\right)
$$

and

$$
\frac{\partial k}{\partial t}=-\frac{1-\gamma}{2} \log (1-\gamma)+\frac{1+\gamma}{2} \log (1+\gamma)-\frac{\gamma}{2} \log \left(2 t \gamma^{4}\right)
$$

Then, if $\frac{\partial k}{\partial \gamma}=\frac{\partial k}{\partial t}=0$, it implies that $\underbrace{\gamma}_{>0}=\underbrace{\log \frac{1-\gamma}{1+\gamma}}_{<0}$. Thus, again the partial derivatives of the function $k$ cannot be both zero.

- Consequently, the maximum of the function $c(\gamma, t)$ is taken at a point $(\gamma, t)$ for which $a(\gamma, t)=$ $b(\gamma, t)$.

This equality gives $(1-\gamma) \log t=\underbrace{1-\gamma-2(1+\gamma) \log (1+\gamma)+\log 4\left(3-\gamma^{2}\right)+\gamma \log \left(2 \gamma^{4}\right)}_{e(\gamma)}$

i.e., $t=\exp \left(\frac{e(\gamma)}{1-\gamma}\right)$. Thus, the function $c(\gamma, t)$ becomes a function $c(\gamma)$. The final computations are done with the Xcas system. We obtain $\gamma_{0}=0.109888 \ldots, t_{0}=12.456245 \ldots$ and $c_{0}=5.895237 \ldots$ as announced in Theorem 2. This finishes the proof.

\section{Remarks:}

1. The constant $t$ obtained for the auxiliary function of Theorem 1 is equal to 15.448160 whereas the optimal value is equal to $t_{0}$.

2. Our final optimisation is done numerically as Serre did. So, as he commented, it would be nice to have an actual proof but the function is too unpleasant for this to be straightforward. 
pol $=[x$,

$x-1$,

$x-2$,

$x^{2}-3 x+1$,

$x^{2}-4 x+2$,

$x^{3}-5 x^{2}+6 x-1$

$x^{3}-6 x^{2}+9 x-1$,

$x^{3}-6 x^{2}+9 x-3$,

$x^{4}-7 x^{3}+14 x^{2}-8 x+1$,

$x^{4}-8 x^{3}+20 x^{2}-17 x+3$,

$x^{4}-8 x^{3}+20 x^{2}-16 x+2$,

$x^{5}-9 x^{4}+27 x^{3}-31 x^{2}+12 x-1$,

$x^{5}-9 x^{4}+28 x^{3}-35 x^{2}+15 x-1$,

$x^{5}-9 x^{4}+27 x^{3}-32 x^{2}+13 x-1$,

$x^{6}-11 x^{5}+44 x^{4}-79 x^{3}+63 x^{2}-18 x+1$,

$x^{6}-11 x^{5}+45 x^{4}-84 x^{3}+70 x^{2}-21 x+1$,

$x^{6}-11 x^{5}+44 x^{4}-78 x^{3}+60 x^{2}-16 x+1$,

$x^{6}-11 x^{5}+44 x^{4}-78 x^{3}+59 x^{2}-15 x+1$,

$2 x^{8}-26 x^{7}+136 x^{6}-367 x^{5}+544 x^{4}-435 x^{3}+171 x^{2}-27 x+1$,

$x^{8}-14 x^{7}+78 x^{6}-221 x^{5}+338 x^{4}-273 x^{3}+106 x^{2}-17 x+1$,

$x^{8}-14 x^{7}+78 x^{6}-222 x^{5}+345 x^{4}-289 x^{3}+120 x^{2}-21 x+1$,

$x^{8}-15 x^{7}+90 x^{6}-276 x^{5}+459 x^{4}-405 x^{3}+171 x^{2}-27 x+1$

$x^{8}-15 x^{7}+90 x^{6}-277 x^{5}+467 x^{4}-428 x^{3}+200 x^{2}-42 x+3$,

$x^{8}-15 x^{7}+91 x^{6}-286 x^{5}+495 x^{4}-462 x^{3}+210 x^{2}-36 x+1$,

$x^{8}-15 x^{7}+90 x^{6}-276 x^{5}+458 x^{4}-400 x^{3}+165 x^{2}-27 x+1$,

$x^{9}-17 x^{8}+120 x^{7}-456 x^{6}+1012 x^{5}-1333 x^{4}+1016 x^{3}-421 x^{2}+86 x-7$,

$x^{9}-17 x^{8}+120 x^{7}-456 x^{6}+1012 x^{5}-1332 x^{4}+1010 x^{3}-409 x^{2}+77 x-5$,

$x^{9}-17 x^{8}+119 x^{7}-444 x^{6}+956 x^{5}-1205 x^{4}+867 x^{3}-335 x^{2}+61 x-4$,

$x^{9}-17 x^{8}+120 x^{7}-456 x^{6}+1011 x^{5}-1324 x^{4}+986 x^{3}-376 x^{2}+57 x-1$,

$x^{9}-17 x^{8}+120 x^{7}-455 x^{6}+1001 x^{5}-1287 x^{4}+924 x^{3}-330 x^{2}+45 x-1$,

$x^{9}-16 x^{8}+104 x^{7}-354 x^{6}+680 x^{5}-745 x^{4}+454 x^{3}-145 x^{2}+21 x-1$,

$x^{10}-18 x^{9}+135 x^{8}-549 x^{7}+1320 x^{6}-1920 x^{5}+1662 x^{4}-813 x^{3}+206 x^{2}-24 x+1$,

$x^{10}-18 x^{9}+136 x^{8}-562 x^{7}+1388 x^{6}-2104 x^{5}+1937 x^{4}-1036 x^{3}+294 x^{2}-36 x+1$,

$x^{10}-18 x^{9}+136 x^{8}-561 x^{7}+1377 x^{6}-2058 x^{5}+1844 x^{4}-941 x^{3}+248 x^{2}-28 x+1$,

$x^{10}-18 x^{9}+136 x^{8}-561 x^{7}+1376 x^{6}-2049 x^{5}+1815 x^{4}-899 x^{3}+220 x^{2}-21 x+1$,

$x^{10}-18 x^{9}+136 x^{8}-562 x^{7}+1387 x^{6}-2096 x^{5}+1913 x^{4}-1002 x^{3}+271 x^{2}-30 x+1$,

$x^{11}-20 x^{10}+171 x^{9}-818 x^{8}+2405 x^{7}-4492 x^{6}+5318 x^{5}-3861 x^{4}+1604 x^{3}-340 x^{2}+32 x-1$,

$x^{11}-21 x^{10}+190 x^{9}-971 x^{8}+3088 x^{7}-6348 x^{6}+8490 x^{5}-7259 x^{4}+3803 x^{3}-1131 x^{2}+166 x-9$,

$x^{11}-21 x^{10}+189 x^{9}-953 x^{8}+2954 x^{7}-5812 x^{6}+7238 x^{5}-5523 x^{4}+2415 x^{3}-538 x^{2}+48 x-1$,

$x^{11}-20 x^{10}+172 x^{9}-832 x^{8}+2485 x^{7}-4733 x^{6}+5730 x^{5}-4261 x^{4}+1812 x^{3}-390 x^{2}+36 x-1$,

$x^{11}-22 x^{10}+208 x^{9}-1106 x^{8}+3635 x^{7}-7643 x^{6}+10286 x^{5}-8609 x^{4}+4194 x^{3}-1040 x^{2}+96 x-2$,

$x^{12}-22 x^{11}+210 x^{10}-1142 x^{9}+3906 x^{8}-8752 x^{7}+12972 x^{6}-12540 x^{5}+7601 x^{4}-2686 x^{3}+491 x^{2}-39 x+1$,

$x^{12}-22 x^{11}+209 x^{10}-1124 x^{9}+3772 x^{8}-8218 x^{7}+11740 x^{6}-10879 x^{5}+6347 x^{4}-2212 x^{3}+421 x^{2}-37 x+1$,

$x^{12}-22 x^{11}+209 x^{10}-1124 x^{9}+3772 x^{8}-8218 x^{7}+11740 x^{6}-10879 x^{5}+6346 x^{4}-2208 x^{3}+417 x^{2}-36 x+1$,

$x^{12}-22 x^{11}+210 x^{10}-1142 x^{9}+3905 x^{8}-8741 x^{7}+12925 x^{6}-12441 x^{5}+7494 x^{4}-2631 x^{3}+481 x^{2}-39 x+1$,

$x^{12}-22 x^{11}+210 x^{10}-1142 x^{9}+3906 x^{8}-8753 x^{7}+12983 x^{6}-12586 x^{5}+7694 x^{4}-2780 x^{3}+534 x^{2}-45 x+1$,

$x^{12}-22 x^{11}+209 x^{10}-1124 x^{9}+3771 x^{8}-8205 x^{7}+11674 x^{6}-10713 x^{5}+6130 x^{4}-2070 x^{3}+380 x^{2}-33 x+1$,

$x^{12}-22 x^{11}+210 x^{10}-1142 x^{9}+3906 x^{8}-8753 x^{7}+12982 x^{6}-12579 x^{5}+7676 x^{4}-2759 x^{3}+523 x^{2}-43 x+1$,

$x^{13}-24 x^{12}+252 x^{11}-1523 x^{10}+5865 x^{9}-15051 x^{8}+26160 x^{7}-30702 x^{6}+23858 x^{5}-11830 x^{4}+3529 x^{3}-575 x^{2}+43 x-1$,

$x^{13}-24 x^{12}+253 x^{11}-1542 x^{10}+6019 x^{9}-15748 x^{8}+28095 x^{7}-34107 x^{6}+27645 x^{5}-14402 x^{4}+4521 x^{3}-766 x^{2}+57 x-1$,

$x^{13}-24 x^{12}+253 x^{11}-1541 x^{10}+6002 x^{9}-15626 x^{8}+27614 x^{7}-32972 x^{6}+26008 x^{5}-12987 x^{4}+3830 x^{3}-596 x^{2}+41 x-1$, $x^{13}-24 x^{12}+253 x^{11}-1542 x^{10}+6020 x^{9}-15762 x^{8}+28176 x^{7}-34359 x^{6}+28106 x^{5}-14913 x^{4}+4862 x^{3}-897 x^{2}+83 x-3$, $x^{13}-23 x^{12}+231 x^{11}-1332 x^{10}+4878 x^{9}-11855 x^{8}+19410 x^{7}-21309 x^{6}+15343 x^{5}-6962 x^{4}+1880 x^{3}-284 x^{2}+24 x-1$, $x^{13}-23 x^{12}+231 x^{11}-1332 x^{10}+4879 x^{9}-11870 x^{8}+19502 x^{7}-21607 x^{6}+15893 x^{5}-7544 x^{4}+2216 x^{3}-376 x^{2}+32 x-1$, $x^{13}-24 x^{12}+253 x^{11}-1541 x^{10}+6002 x^{9}-15626 x^{8}+27615 x^{7}-32981 x^{6}+26039 x^{5}-13038 x^{4}+3871 x^{3}-611 x^{2}+43 x-1$, $x^{13}-23 x^{12}+233 x^{11}-1368 x^{10}+5153 x^{9}-13021 x^{8}+22425 x^{7}-26231 x^{6}+20423 x^{5}-10189 x^{4}+3065 x^{3}-505 x^{2}+39 x-1$, $x^{13}-24 x^{12}+252 x^{11}-1523 x^{10}+5866 x^{9}-15066 x^{8}+26251 x^{7}-30991 x^{6}+24378 x^{5}-12369 x^{4}+3847 x^{3}-679 x^{2}+60 x-2$, $x^{14}-25 x^{13}+277 x^{12}-1794 x^{11}+7543 x^{10}-21627 x^{9}+43227 x^{8}-60517 x^{7}+58789 x^{6}-38703 x^{5}+16577 x^{4}-4331 x^{3}+$ $623 x^{2}-42 x+1$,

$x^{14}-26 x^{13}+299 x^{12}-2004 x^{11}+8684 x^{10}-25520 x^{9}+51915 x^{8}-73349 x^{7}+71240 x^{6}-46462 x^{5}+19566 x^{4}-4998 x^{3}+$ $699 x^{2}-45 x+1$

$x^{14}-25 x^{13}+276 x^{12}-1774 x^{11}+7370 x^{10}-20778 x^{9}+40621 x^{8}-55317 x^{7}+52002 x^{6}-33027 x^{5}+13693 x^{4}-3531 x^{3}+$ $526 x^{2}-39 x+1$,

$x^{15}-27 x^{14}+325 x^{13}-2303 x^{12}+10687 x^{11}-34185 x^{10}+77283 x^{9}-124595 x^{8}+142812 x^{7}-114758 x^{6}+62982 x^{5}-22638 x^{4}+$ $4986 x^{3}-605 x^{2}+35 x-1$,

$x^{16}-30 x^{15}+406 x^{14}-3279 x^{13}+17620 x^{12}-66499 x^{11}+181381 x^{10}-362482 x^{9}+532494 x^{8}-571922 x^{7}+442965 x^{6}-$ $241724 x^{5}+89680 x^{4}-21412 x^{3}+3008 x^{2}-211 x+5$,

$x^{17}-31 x^{16}+435 x^{15}-3657 x^{14}+20548 x^{13}-81514 x^{12}+235128 x^{11}-500454 x^{10}+789441 x^{9}-919189 x^{8}+780461 x^{7}-$ $473293 x^{6}+198761 x^{5}-55359 x^{4}+9643 x^{3}-971 x^{2}+50 x-1$,

$x^{19}-34 x^{18}+530 x^{17}-5022 x^{16}+32335 x^{15}-149817 x^{14}+516044 x^{13}-1346309 x^{12}+2685700 x^{11}-4107313 x^{10}+4799740 x^{9}-$ $4247503 x^{8}+2803746 x^{7}-1349973 x^{6}+459380 x^{5}-105694 x^{4}+15446 x^{3}-1312 x^{2}+57 x-1$,

$x^{19}-35 x^{18}+561 x^{17}-5459 x^{16}+36045 x^{15}-170995 x^{14}+602002 x^{13}-1602180 x^{12}+3253797 x^{11}-5055385 x^{10}+5990265 x^{9}-$ $5367784 x^{8}+3587201 x^{7}-1752386 x^{6}+608784 x^{5}-144820 x^{4}+22364 x^{3}-2074 x^{2}+102 x-2$,

$x^{19}-35 x^{18}+562 x^{17}-5490 x^{16}+36480 x^{15}-174653 x^{14}+622572 x^{13}-1683908 x^{12}+3490140 x^{11}-5560315 x^{10}+6790955 x^{9}-$ 
$6306710 x^{8}+4391990 x^{7}-2246607 x^{6}+819883 x^{5}-205010 x^{4}+33197 x^{3}-3210 x^{2}+162 x-3$,

$x^{19}-34 x^{18}+530 x^{17}-5022 x^{16}+32335 x^{15}-149818 x^{14}+516067 x^{13}-1346543 x^{12}+2687089 x^{11}-4112655 x^{10}+4813716 x^{9}-$ $4272910 x^{8}+2835949 x^{7}-1378113 x^{6}+475895 x^{5}-111922 x^{4}+16852 x^{3}-1480 x^{2}+65 x-1$,

$x^{19}-35 x^{18}+560 x^{17}-5432 x^{16}+35716 x^{15}-168601 x^{14}+590399 x^{13}-1562609 x^{12}+3155931 x^{11}-4876919 x^{10}+5748184 x^{9}-$ $5122367 x^{8}+3400560 x^{7}-1645505 x^{6}+562769 x^{5}-130255 x^{4}+19170 x^{3}-1634 x^{2}+69 x-1$,

$x^{19}-35 x^{18}+561 x^{17}-5460 x^{16}+36072 x^{15}-171322 x^{14}+604350 x^{13}-1613309 x^{12}+3290472 x^{11}-5141552 x^{10}+6135753 x^{9}-$ $5543184 x^{8}+3734798 x^{7}-1835017 x^{6}+636489 x^{5}-148716 x^{4}+21848 x^{3}-1820 x^{2}+73 x-1$,

$x^{21}-38 x^{20}+667 x^{19}-7177 x^{18}+52983 x^{17}-284588 x^{16}+1151004 x^{15}-3578829 x^{14}+8659711 x^{13}-16405967 x^{12}+24365366 x^{11}-$ $28282682 x^{10}+25479854 x^{9}-17617116 x^{8}+9200833 x^{7}-3552227 x^{6}+984814 x^{5}-188443 x^{4}+23524 x^{3}-1758 x^{2}+68 x-1$, $x^{21}-38 x^{20}+667 x^{19}-7176 x^{18}+52953 x^{17}-284180 x^{16}+1147673 x^{15}-3560605 x^{14}+8589071 x^{13}-16205933 x^{12}+23944730 x^{11}-$ $27621392 x^{10}+24703168 x^{9}-16940663 x^{8}+8770162 x^{7}-3356234 x^{6}+923145 x^{5}-175690 x^{4}+21932 x^{3}-1657 x^{2}+66 x-1$, $x^{21}-38 x^{20}+667 x^{19}-7177 x^{18}+52983 x^{17}-284589 x^{16}+1151029 x^{15}-3579108 x^{14}+8661547 x^{13}-16413896 x^{12}+24389035 x^{11}-$ $28332790 x^{10}+25555808 x^{9}-17699308 x^{8}+9263369 x^{7}-3584683 x^{6}+995713 x^{5}-190600 x^{4}+23733 x^{3}-1764 x^{2}+68 x-1$, $x^{22}-41 x^{21}+780 x^{20}-9141 x^{19}+73882 x^{18}-436925 x^{17}+1957369 x^{16}-6785074 x^{15}+18431384 x^{14}-39495678 x^{13}+$ $66883162 x^{12}-89298078 x^{11}+93411216 x^{10}-75773874 x^{9}+46973720 x^{8}-21823973 x^{7}+7409684 x^{6}-1780366 x^{5}+290753 x^{4}-$ $30684 x^{3}+1958 x^{2}-68 x+1$,

$x^{26}-47 x^{25}+1036 x^{24}-14237 x^{23}+136775 x^{22}-976348 x^{21}+5373366 x^{20}-23351006 x^{19}+81411511 x^{18}-230121322 x^{17}+$ $530874984 x^{16}-1003098910 x^{15}+1554044082 x^{14}-1971645439 x^{13}+2041748237 x^{12}-1716463523 x^{11}+1162576984 x^{10}-$ $\left.628064340 x^{9}+267174439 x^{8}-88044247 x^{7}+22012762 x^{6}-4064156 x^{5}+534378 x^{4}-47549 x^{3}+2650 x^{2}-81 x+1\right]$

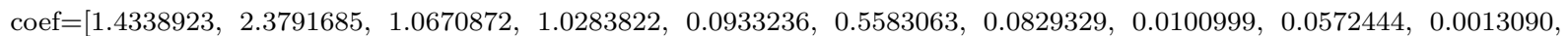
$0.0007186,0.0849735,0.1862799,0.0303978,0.0045486,0.1091599,0.0038395,0.0088677,0.0025727,0.0036384,0.0062433$, $0.0026132,0.0017400,0.0170124,0.0015320,0.0000021,0.0037592,0.0022086,0.0077010,0.0044824,0.0024851,0.0196657$, $0.0455162,0.0039525,0.0001574,0.0111271,0.0026080,0.0025693,0.0016778,0.0048469,0.0022862,0.0117032,0.0126292$, $0.0013425,0.0037429,0.0063653,0.0048968,0.0019928,0.0185382,0.0105638,0.0024603,0.0021767,0.0014564,0.0027321$, 0.0147307, 0.0038494, 0.0107454, 0.0034055, 0.0011166, 0.0014229, 0.0003166, 0.0053721, 0.0036493, 0.0012079, 0.0022056, 0.0017564, 0.0017591, 0.0036141, 0.0016779, 0.0146680, 0.0053908, 0.0043565, 0.0022349, 0.0009102]; 


\section{References}

[ABP] J. Aguirre, M. Bilbao, J. C. Peral. The trace of totally positive algebraic integers, Math. Comp.,75 (2006), no 253, 385-393.

[AP1] J. Aguirre and J.C. Peral. The Trace Problem for Totally Positive Algebraic Integers, Number Theory and Polynomials. (Conference proceedings, University of Bristol, 3-7 April 2006, editors J.F. McKee and C.J. Smyth). LMS Lecture notes

[AP2] J. Aguirre and J.C. Peral. The integer Chebyshev constant of Farey intervals, Publ. Mat. 2007, Proceedings of the Primeras Jornadas de Teoría de Números, 11-27.

[AP3] J. Aguirre and J.C. Peral. The trace problem for totally positive algebraic integers. With an appendix by Jean-Pierre Serre, London Math. Soc. Lecture Note Ser., 352, Number theory and polynomials, 1-19, Cambridge Univ. Press, Cambridge, 2008.

[DW] X. Dong and Q. Wu The absolute trace of totally positive reciprocal algebraic integers, J. Number Theory 170 (2017), 66-74.

[F1] V.Flammang. Une nouvelle minoration pour la trace absolue des entiers algébriques totalement positifs available at: http://arxiv.org/abs/1907.09407, (2019).

[LW] Y. Liang and Q. Wu. The trace problem for totally positive algebraic integers, J. Aust. Math. Soc. 90 (2011), no. 3, 341-354.

[Sc] I. Schur. Uber die Verteilung der Wurzeln bei gewissen algebraischen Gleichungen mit ganzzahligen Koeffizienten, Math. Z. 1 (1918), 377-402.

[Si] C.L. Siegel. The trace of totally positive and real algebraic integers, Ann. of Maths, 46 (1945), 302-312.

[S1] C.J. Smyth. The mean value of totally real algebraic numbers, Math. Comp. 42 (1984), 663-681.

[S2] C.J. Smyth. Totally positive algebraic integers of small trace, Ann. Inst. Fourrier, Grenoble 33 (1984), 1-28.

[Wu] Q. Wu. On the linear independence measure of logarithms of rational numbers, Math. Comp. 72 (2003),901-911.

UMR CNRS 7502. IECL, Université de Lorraine, site de Metz, Département de Mathématiques, UFR MIM,

3 rue Augustin Fresnel BP 4511257073 Metz cedex 3 E-mail address : valerie.flammang@univlorraine.fr 